\title{
Time course and specificity of sensory-motor alpha modulation during the observation of hand motor acts and gestures: a high density EEG study
}

\author{
Alena Streltsova - Cristina Berchio • \\ Vittorio Gallese • Maria Alessandra Umilta'
}

Received: 9 February 2010/ Accepted: 14 July 2010/Published online: 3 August 2010

(c) The Author(s) 2010. This article is published with open access at Springerlink.com

\begin{abstract}
The main aim of the present study was to explore, by means of high-density EEG, the intensity and the temporal pattern of event-related sensory-motor alpha desynchronization (ERD) during the observation of different types of hand motor acts and gestures. In particular, we aimed to investigate whether the sensory-motor ERD would show a specific modulation during the observation of hand behaviors differing for goal-relatedness (hand grasping of an object and meaningless hand movements) and social relevance (communicative hand gestures and grasping within a social context). Time course analysis of alpha suppression showed that all types of hand behaviors were effective in triggering sensory-motor alpha ERD, but to a different degree depending on the category of observed hand motor acts and gestures. Meaningless gestures and hand grasping were the most effective stimuli, resulting in the strongest ERD. The observation of social hand behaviors such as social grasping and communicative gestures, triggered a more dynamic time course of ERD compared to that driven by the observation of simple grasping and meaningless gestures. These findings indicate that the observation of hand motor acts and gestures evoke the activation of a motor resonance mechanism that differs on the basis of the goal-relatedness and the social relevance of the observed hand behavior.
\end{abstract}

\footnotetext{
A. Streltsova · C. Berchio · V. Gallese · M. A. Umilta' ( $₫)$ Department of Neuroscience, Section of Physiology, University of Parma, Via Volturno 39, 43100 Parma, Italy e-mail: mariaalessandra.umilta@unipr.it

V. Gallese · M. A. Umilta'

IIT (Italian Institute of Technology) Brain Center

for Social and Motor Cognition, Parma, Italy
}

Keywords EEG $\cdot$ Sensory-motor alpha . Mirror mechanism · Motor resonance .

Social context . Time course

\section{Introduction}

Gastaut and co-workers first described the desynchronization of an EEG rhythm within the alpha band $(8-13 \mathrm{~Hz})$, recorded from central electrodes, occurring not only during the execution of active movements, but also during their observation (Cohen-Seat et al. 1954; Gastaut and Bert 1954). This result was confirmed many years later by Cochin et al. (1998, 1999), Altschuler et al. (1997, 2000) using EEG recordings, and by Hari et al. (1998) using magnetoencephalography (MEG). In particular, Hari and co-workers demonstrated that the desynchronization during action observation includes rhythms originating from the cortex inside the central sulcus (Hari and Salmelin 1997; Salmelin and Hari 1994, for a review, see Pineda 2005).

It has been proposed that the suppression of the sensorymotor alpha rhythm, also termed as event-related desynchronization (ERD), during action execution and observation, could be due to a neurophysiological mechanism of motor resonance, the mirror mechanism, likely due to the activation of neurons endowed with properties similar to those of mirror neurons, discovered in the premotor and posterior parietal cortices of macaque monkeys. Mirror neurons discharge both during the execution of goal-related motor acts and during their observation when executed by others (Di Pellegrino et al. 1992; Gallese et al. 1996; Rizzolatti et al. 1996).

A growing body of research showing that a number of human cortical areas are activated by both action execution and observation supports the existence of a mirror 
mechanism in humans. These cortical areas include the lower part of precentral gyrus, the posterior part of inferior frontal gyrus (IFG) and the rostral part of the inferior parietal lobule (for a review, see Rizzolatti and Craighero 2004; Gallese et al. 2004; Cattaneo and Rizzolatti 2009). Recently, evidence at the single neuron-level about the existence of the mirror mechanism in the human brain was provided (Mukamel et al. 2010).

Several neuroimaging studies have reported the activation of distinct cortical regions within premotor and posterior parietal cortices during the observation/execution of goal-related hand, mouth and foot actions (Buccino et al. 2001; Aziz-Zadeh et al. 2006) during the observation/ execution of meaningless hand movements (Lui et al. 2008; Villarreal et al. 2008) during the execution of a noisy action and listening to the corresponding sound (Gazzola et al. 2006). Few studies have demonstrated communicative face and hand actions showing an activation of cortical regions endowed with mirror properties (Nakamura et al. 1999; Nakamura et al. 2004; Montgomery et al. 2007; Villarreal et al. 2008).

Recent EEG studies of the mirror mechanism have shown that the observation and execution of a motor act is accompanied by an event-related alpha desynchronization (ERD) reflected in a relative decrease in power of sensorymotor alpha $(8-13 \mathrm{~Hz})$ and beta $(13-30 \mathrm{~Hz})$ frequency bands (Hari 2006). More generally, ERD reflects a cortical deactivation which depends on the task and it is usually measured with respect to the baseline that contains a clear peak in the frequency band of interest (Pfurtscheller and Lopes da Silva 1999; Klimesch 1999). It has been shown that during movement execution ERD reflects an inhibition of the motor cortex and has a pre-movement onset. (Pfurtscheller and Lopes da Silva 1999; Calmels et al. 2006 for review, see Pineda 2005). Several EEG studies have shown sensory-motor alpha ERD during the observation of different types of grasping (Muthukumaraswamy et al. 2004; Muthukumaraswamy and Johnson 2004a; Perry and Bentin 2009), meaningless gestures (Babiloni et al. 2002) and sequential finger movements (Calmels et al. 2006). Recent EEG studies aiming to investigate sensory-motor alpha suppression during the observation of more complex stimuli such as social interaction scenes, body movements from different perspectives and dance performance, have shown that alpha ERD can be modulated by the degree of social relevance (Kilner et al. 2006; Oberman et al. 2007b) and by the motor expertise of the observer (Orgs et al. 2008; Babiloni et al. 2009). Furthermore, recent findings demonstrate the existence of an observation/execution matching system even in infants and young children (Lepage and Théoret 2006; Southgate et al. 2009, 2010).

The main aim of the present study was to explore, by means of high-density EEG, the sensitivity of sensory- motor ERD during the observation of different types of hand motor acts and gestures. In particular, we aimed to investigate the specificity of sensory-motor ERD for processing hand behaviors differing along two dimensions: their goal-relatedness and social relevance

All participants also performed a control motor execution task. Similarly to other studies (Muthukumaraswamy and Johnson 2004a; Southgate et al. 2009, 2010), the aim of the motor task (key-press) was to identify the frequency band that was functionally related to motor activation in each individual participant.

Since the neural basis of the mirror mechanism is the presence of a parieto-premotor neural circuit activated during both motor execution and observation, most likely due to the presence of neurons instantiating functional properties similar to those of macaque monkey mirror neurons (Gallese et al. 1996; Rizzolatti et al. 1996) we measured sensory-motor ERD in the observation task by considering the same frequencies that were attenuated during the execution task. In addition, and more importantly, the temporal course of alpha suppression was carefully investigated.

\section{Methods}

\section{Participants}

Thirteen right-handed healthy volunteers (7 males, 6 females, mean age $27 \pm 7$ years old) participated in the experiment. Participants were recruited by public announcement and were blind to the experimental goals. All participants were right-handed as assessed by the Edinburgh Handedness Inventory (Oldfield 1971). None of them reported the presence of any neurological or psychiatric disorder and had normal or corrected to normal vision. Before the experiment, all participants received written experimental instructions. Informed consent was obtained from all participants before entering the study. The study was approved by the local Ethical Committee.

\section{Experimental procedure}

Participants were seated comfortably in front of a computer monitor used for stimuli presentation, located at a distance of $60 \mathrm{~cm}$. To minimize participants' movements during the experiment, they were asked to keep their arms on a table in front of them and to stay as motionless and relaxed as possible. The experiment included observation and execution tasks.

Each execution trial started with a grey background with a varying duration of 6 to $9 \mathrm{~s}$ randomized across trials (baseline) followed by two fixation crosses presented for $500 \mathrm{~ms}$ in the middle of the screen. Participants were asked 
to press a key with their right index finger as soon as the fixation crosses were replaced by a black screen, and then to return the hand to the initial position. Each observation trial started with a grey background with a varying duration of 6 to $9 \mathrm{~s}$ randomized across trials (baseline) followed by a single fixation cross presented for $500 \mathrm{~ms}$ in the middle of the screen and then by the presentation of video clips. The single cross indicated to participants that they should passively observe the visual stimuli presented after the cross disappeared (see Fig. 1 for experimental paradigm). Stimuli consisted of four categories of video clips showing: (a) a hand grasping an object positioned on a table, and then holding it; (b) a hand grasping the same object positioned on the palm of the hand of another individual, and then holding it; (c) meaningless hand movements; (d) communicative hand gestures. All trials and conditions were presented in randomized order and were randomized across participants. In order to maintain participants' attention, they were told that they would be asked about the content of the movies at the end of the experiment.

Stimuli characteristics and amount of movement analysis

Videos were in .avi format and were recorded by a digital camera with a resolution of $720 \times 576$ color pixels at a frame rate of 25 frames/s positioned at $1.5 \mathrm{~m}$ distance from an actor. Each video clip was $3 \mathrm{~s}$ long (frequency rate: $25 \mathrm{~Hz}, 75$ frames, pixel aspect ratio: 1.067) and was presented at a distance of $60 \mathrm{~cm}$ from the participants. The video clips showed the right hand of one of two female actors from an allocentric point of view. Each category of stimuli included two different video clips and the total number of stimuli was 16,8 video clips for each actor (see Fig. 2). In meaningless movements condition participants observed a thumb moving towards and away from the palm and an opening and closing hand. Communicative gestures category consisted of thumbs up "Good" and of a typical Italian hand gesture with the wrist and the forearm moving back and forward with the fingers closed, meaning "What are you saying?" In grasping condition participants observed a whole-hand grasping (hand grasping a large sphere from the table) and a precision grip (hand grasping a small sphere from the table). Similarly, in social grasping condition participants observed a hand grasping a large sphere from the palm of another individual and a hand grasping a small sphere from the palm of another individual. Hand behaviours happened once in each video-clip in all experimental conditions. For the offline analysis of the quantity of movement being observed during the different time-bins of the stimuli, three digital markers were put in each frame of all video clips on the thumb, index finger and palm of the actors' hand. Videos were subsequently examined frame by frame using a tracking program. Files with markers coordinates were exported in order to average the amount of movement in each time-bin. For each video clip the total amount of movement was calculated as the average distance between initial and final positions of the three markers in each of the $3 \mathrm{~s}$ of the video clips. For statistical analysis, mean distances in each stimuli category were multiplied by the minimum number of trials used for each condition in the subsequent EEG data analysis. Paired t-tests were carried out in order to compare the above parameters across three time intervals in the four experimental conditions.

\section{EEG recording}

EEG data were acquired by a 128-channel Sensor Net (Electrical Geodesic, Eugene, USA) and recorded within
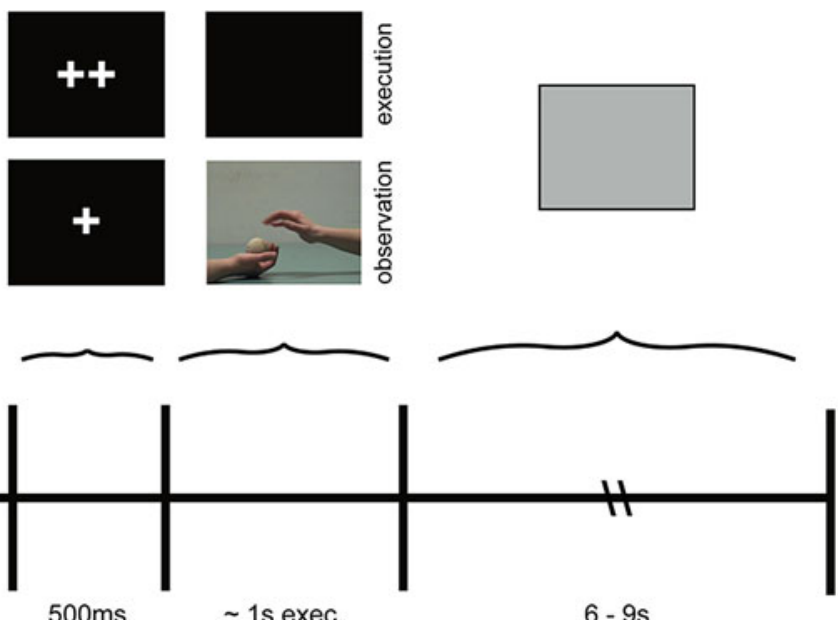

$6-9 s$

$500 \mathrm{~ms}$

$\sim 1$ s exec.

$6-9 s$

3s obs.

Baseline

Fixation

Task

Baseline 

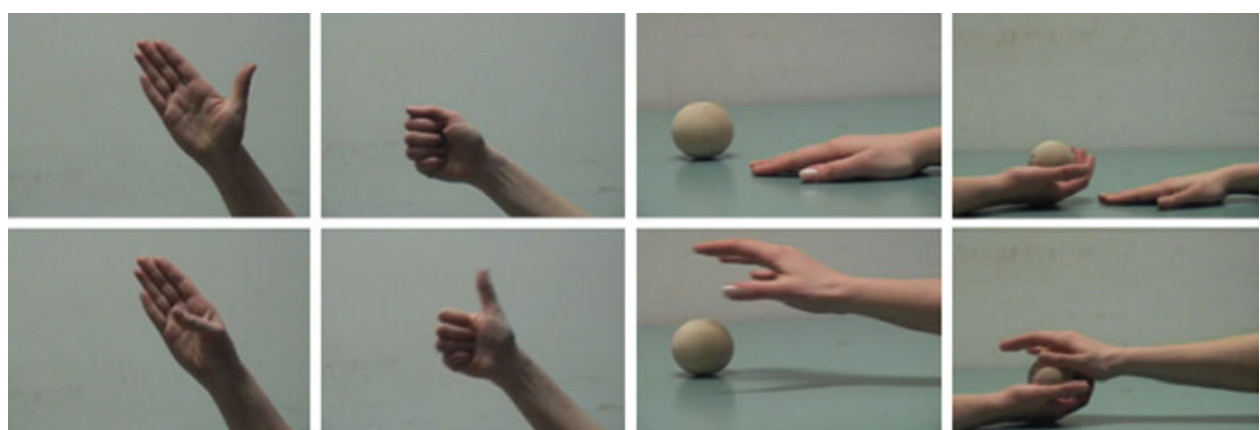

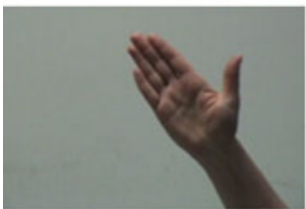

Meaningless

Movement

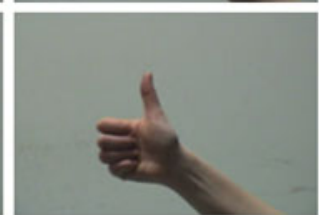

Communicative Gesture

Fig. 2 Examples of frames extracted from used video clips. Four categories of stimuli were employed and each category consisted of two different stimuli. (1) Meaningless movements: thumb moving towards and away from the palm; opening/closing the hand; (2) communicative gestures: thumbs up "Good"; typical Italian hand gesture "What are you saying?"; (3) grasping: whole hand (hand

standard EGI package Net Station 4.3.1. EEG was sampled at $250 \mathrm{~Hz}$ and band-pass filtered at $0.3-100 \mathrm{~Hz}$, electrodes impedance was less than $100 \mathrm{~K} \Omega$. The raw EEG data were recorded with the vertex $(\mathrm{Cz})$ as the online reference and re-referenced off-line to the common average (Muthukumaraswamy et al. 2004).

Stimuli were presented with E-Prime 2.0. and, at the beginning of each trial, all event markers were sent to Net Station. The experiment took place in an isolated and lit room, the stimuli were presented on a 17 -in. computer monitor. Participants' motion was monitored by the experimenter and video-recorded for off-line analysis; if participants moved during the observation or rest conditions, the trial was excluded from further data analysis.

\section{EEG data analysis}

EEG data were filtered off-line with band-pass filter $0.3-30 \mathrm{~Hz}$ and segmented into certain time epochs. In the observation and execution tasks 3,000 ms of grey screen before the appearance of the fixation crosses were taken as a baseline. Execution trials were segmented into 2,000 ms epochs which started $1,500 \mathrm{~ms}$ before the motor response and ended $500 \mathrm{~ms}$ after it. Only the trials in which participants responded correctly were analysed. The observation trials were segmented into $3,000 \mathrm{~ms}$ epochs that corresponded to the whole duration of the presented video clips. The trials in which participants produced eye-blinks

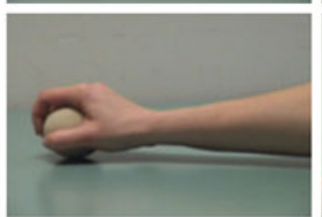

Grasping

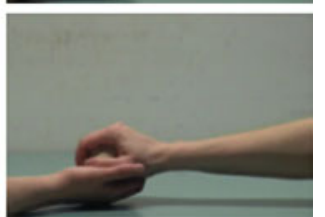

Social Grasping

grasping a large sphere from the table); precision grip (hand grasping a small sphere from the table); (4) social grasping: whole hand (hand grasping a large sphere from the palm of another individual); precision grip (hand grasping a small sphere from the palm of another individual)

and movement artefacts were rejected on the basis of the artefacts detection tool supplied by Net Station and on the basis of a subsequent careful visual inspection of each segment. A minimum number of 20 trials was kept in each condition; two participants who had less trials were excluded from further data analysis.

The time-frequency analysis was performed by continuous Morlet wavelet transformation in $0.5 \mathrm{~Hz}$ intervals in the frequency range from 1 to $30 \mathrm{~Hz}$. Frequency-power coefficients were calculated by taking the average across trials. The wavelet transformation was calculated separately for each participant in all 128 channels for each condition.

Statistical analysis was performed on a selected cluster of 8 electrodes in each hemisphere located around standard $\mathrm{C} 3$ and $\mathrm{C} 4$ sites. The same cluster was used in a previous study that demonstrated the desynchronization of sensory-motor alpha activity during the observation of hand motor acts (Muthukumaraswamy and Johnson 2004a, b; Muthukumaraswamy et al. 2004; Bernier et al. 2007).

Determination of participants' specific frequency bands and statistical analysis

For each participant specific alpha-frequency bands were selected in the range $8-14 \mathrm{~Hz}$ following the procedure described in previous studies (Oberman et al. 2007a, b; Babiloni et al. 2009). The individual peak (F) of attenuated frequency was determined by calculating a ratio 
between the frequency power in the execution and in the baseline conditions in the six following sub-frequency bands: 8-9, 9-10, 10-11, 11-12, 12-13, 13-14 Hz. Each value was then transformed into a log-ratio and the frequency which corresponded to log-ratio with the most negative value was taken as F. A $3 \mathrm{~Hz}$ range frequency band was chosen for each participant: the interval $(\mathrm{F}-1$; $\mathrm{F}+1)$ in which a lower frequency power was revealed in execution compared to the baseline condition. For the following statistical analyses, the frequency power in this $3 \mathrm{~Hz}$ range was extracted in all conditions (see Table 1 and Fig. 3).

The data used for statistical analysis in the execution task were extracted from two different time intervals: $500 \mathrm{~ms}$ before (movement interval 1) and $500 \mathrm{~ms}$ after key press (movement interval 2). In order to assess alpha desynchronization during execution with respect to baseline, we performed a repeated measures $2 \times 3$ ANOVA with the main factors of Hemisphere (left vs. right) and Condition (baseline, movement interval 1, movement interval 2). The data used for statistical analysis in the observation task were extracted from 3 time intervals corresponding to the first, the second, and the third

Table $13 \mathrm{~Hz}$ wide frequency bands, within 8-14 Hz range, selected for each participant on the basis of movement execution

\begin{tabular}{ll}
\hline Selected frequency band $(\mathrm{Hz})$ & Participant No. \\
\hline $8-11$ & $1,3,7,8,11$ \\
$9-12$ & $2,4,10$ \\
$10-13$ & 9 \\
$11-14$ & 5,6 \\
\hline
\end{tabular}

The selected frequency bands were used for the statistical analysis in observation task
$1000 \mathrm{~ms}$ of each video clip. The $3000 \mathrm{~ms}$ of baseline were also subdivided in 3 time intervals of $1000 \mathrm{~ms}$ each. A $2 \times 5 \times 3$ repeated measures ANOVA was performed with 5 levels of Condition (baseline, grasping, social grasping, meaningless movement, communicative gesture), 2 levels of Hemisphere (left vs. right) and 3 levels of Time (second 1, 2, and 3). Post-hoc analysis (Fisher test) was applied on all significant main factors and interactions.

Since the sensory-motor alpha frequency band $(8-14 \mathrm{~Hz})$ overlaps with the posterior alpha band, it is possible that recordings in central areas might be affected by this posterior activity. In order to check whether the sensorymotor alpha recorded in central areas, was affected by posterior alpha, we performed an additional analysis in occipital (O1 and $\mathrm{O} 2$ ) and central (C3 and $\mathrm{C} 4)$ electrodes. The log-ratio of the frequency-power was calculated between the baseline (grey screen) and an additional passive condition, performed at the beginning of the experimental session, in which participants kept their eyes closed for $60 \mathrm{~s}$. No alpha suppression was revealed at central electrodes, indicated by zero value of log-ratio. In the occipital sites however, negative logratio was found showing a higher alpha power in the eyes-closed condition compared to baseline. Therefore, in further data analysis we presumed that sensory-motor alpha modulation in the observation task was not related to mere visual processing of stimuli. Figure 4 shows time frequency plots of the voltage amplitude, of the entire head of all participants, in the baseline and in the average of all the observation conditions. It is clear that the amplitude decreases in the central regions during the observation conditions with respect to the baseline. This decrease is much less pronounced in the occipital region.
Fig. 3 Sensory-motor alpha modulation during movement execution (key press). Figure represents the extracted EEG frequency-power from C3 electrode of one participant's data in $9-12 \mathrm{~Hz}$ band of alpha frequency range

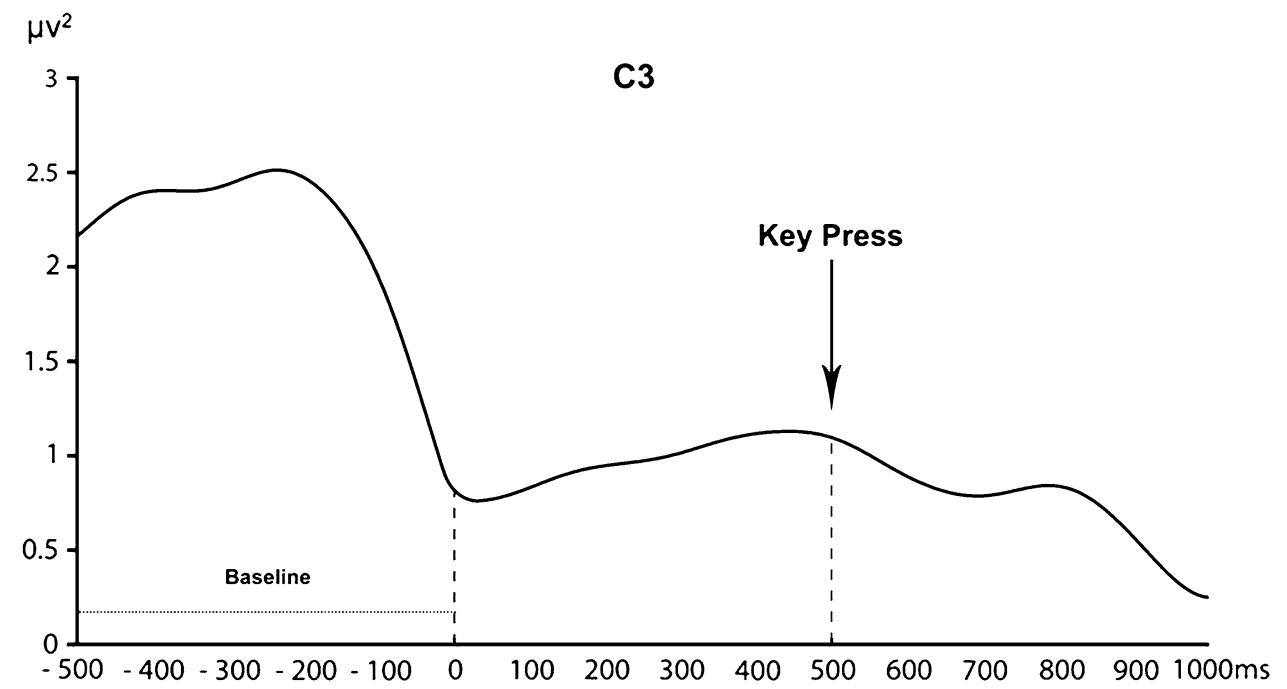




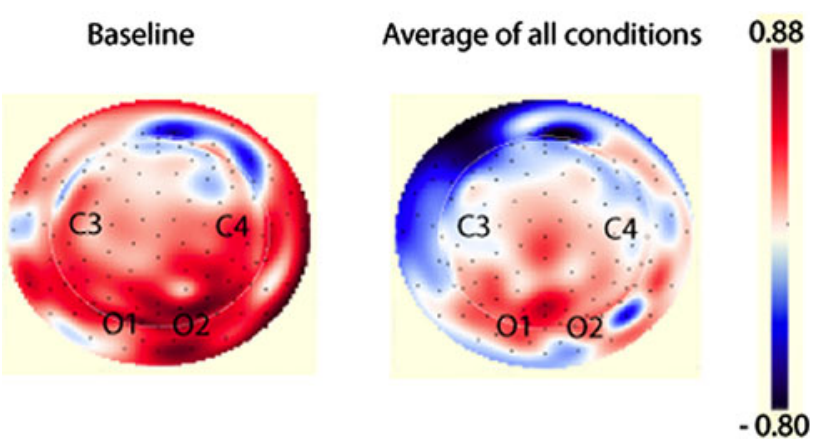

Fig. 4 Time frequency plots of the voltage amplitude, in the baseline (left panel) and in the average of all the observation conditions (right panel), recorded over the entire head of all participants. Amplitudes are represented over the entire surface of the head. The amplitude scale defines the voltage value of different points on the map by a color spectrum

\section{Results}

\section{Execution}

In order to assess alpha desynchronization in central sensory-motor areas during the execution of a motor act, we compared the frequency power extracted from wavelet in the baseline condition with that in the $500 \mathrm{~ms}$ before and $500 \mathrm{~ms}$ after key press (see "Methods"). The results of the repeated measures $2 \times 3$ ANOVA showed a main effect of Condition $(P<0.0001)$. A post-hoc Fisher test revealed that the two movement intervals were significantly different from baseline (both $P_{\mathrm{s}}<0.0001$ ) and that the two movement periods differed, with a greater desynchronization during the second movement interval (see Fig. 3).

\section{Observation}

In order to assess alpha desynchronization in central sensory-motor areas during the four observation conditions, we compared the frequency power extracted from wavelet in the baseline condition with that during observation (see Fig. 5).

A $2 \times 5 \times 3$ ANOVA design was created with 5 levels of Condition (baseline, grasping, social grasping, meaningless movement, communicative gesture), 2 levels of Hemisphere (right vs. left), 3 levels of Time (second 1, 2 and 3, see "Methods"). The results of the ANOVA revealed a significant main effect of Condition $(P<0.01)$ as well as a significant condition $\times$ time interaction $(P<0.05)$. Post-hoc comparisons (Fisher test) were
Fig. 5 Figures represent EEG time-frequency spectrum for one participant extracted from C3 site in the four observation conditions and in the baseline. A continuous wavelet transformation was performed for the frequencies from 1 to $30 \mathrm{~Hz}$ in a time window of $3 \mathrm{~s}$, that corresponds to the total duration of video clips. Red colour indicates an increased synchronization (ERS) while blue colour indicates a decreased synchronization (ERD) in a given frequency band (see scale bar). The orange line added to each panel highlights the $3 \mathrm{~Hz}$ wide frequency band $(9-12 \mathrm{~Hz})$, within the alpha range, used for statistical analysis for this participant
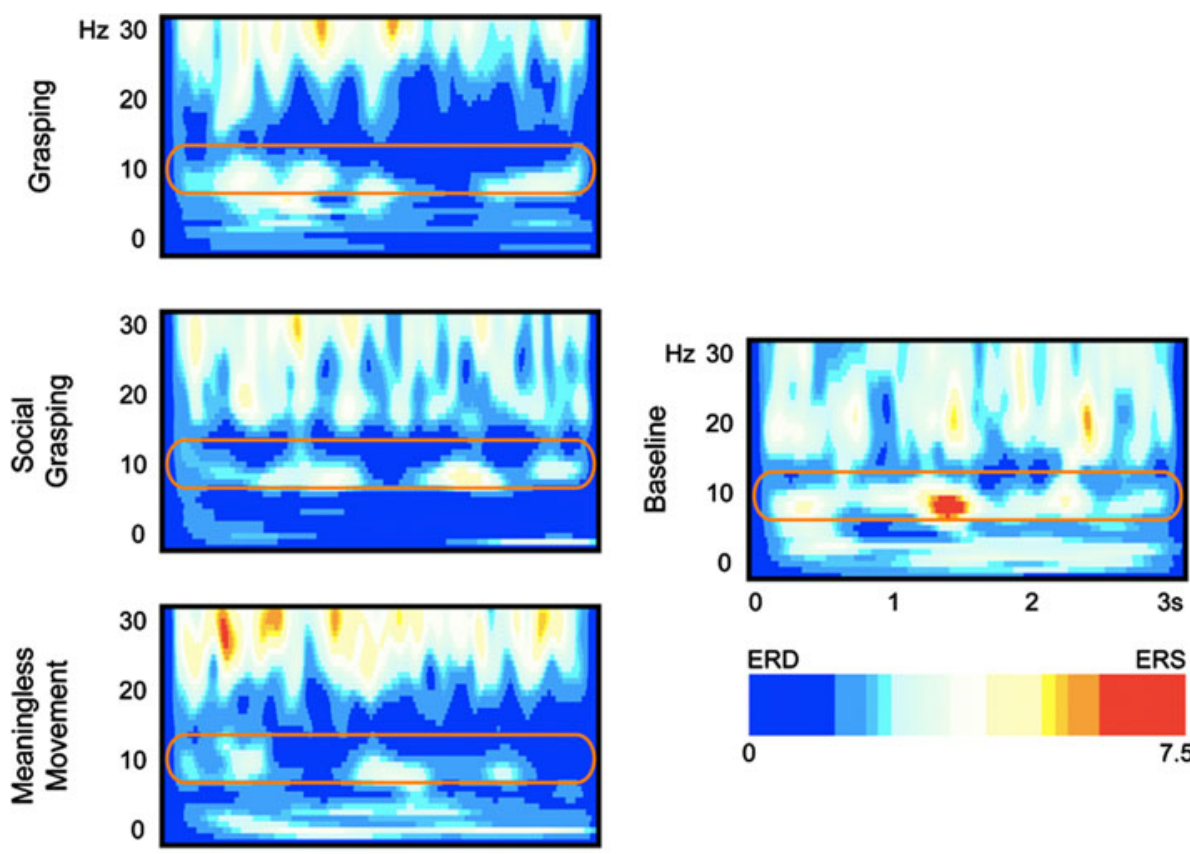

ERD

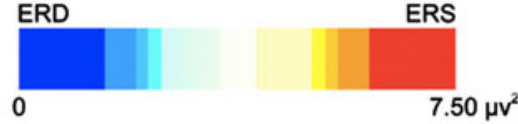

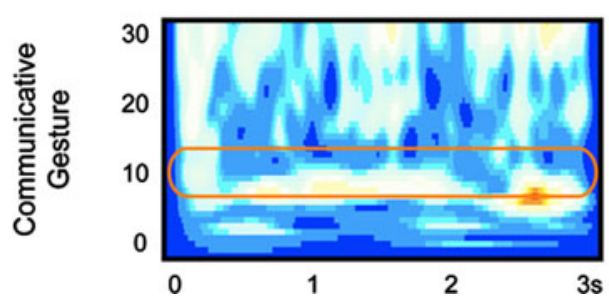


performed to further explore the Condition effect. A significant desynchronization, with respect to baseline, was revealed in all observation conditions $\left(P_{\mathrm{s}}<0.05\right)$, except communicative gestures $(P=0.09)$. The amplitude of ERD was not significantly different $\left(P_{\mathrm{s}}>0.05\right)$ among the three observation conditions showing significant desynchronization.

As far as the condition $\times$ time interaction was concerned, all conditions showed a significant ERD with respect to baseline in the three time intervals $\left(P_{\mathrm{s}}<0.05\right)$ with the exception of communicative gestures in the second time interval (see Fig. 6). Thus, although all 4 types of observed movements induced a significant desynchronization, they differed in the ERD temporal pattern.

The comparison among the three time intervals within each condition revealed that during the observation of grasping and meaningless gestures there was no significant difference in the amplitude of ERD $(P>0.05)$ throughout the entire duration of the video clips. Within these two conditions, sensory-motor alpha suppression showed the same intensity in all of the three time intervals. During the observation of communicative gestures only the first and the third time intervals showed ERD, which was greater during the first $1000 \mathrm{~ms}$ compared to the third time-bin $(P<0.005)$. During the observation of social grasping all time intervals showed a significant desynchronization with respect to the baseline. However, the first and the third time

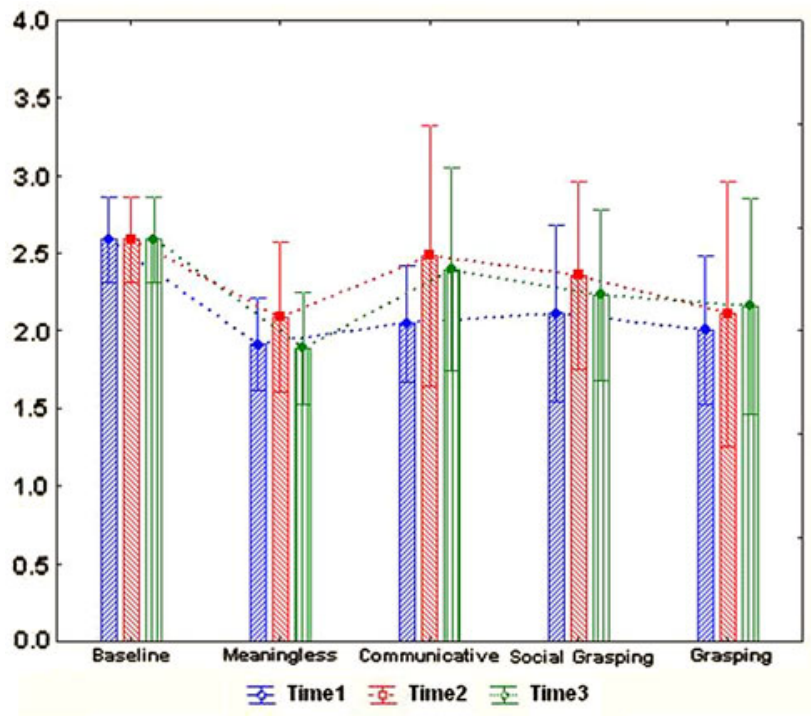

Fig. 6 ERD intensity and time course during baseline, observation of motor acts and gestures. Plots represent the significant condition $\times$ time interaction $(F(8,80)=2.16, P=0.039)$ resulted from an ANOVA (see methods for details) performed on the averaged EEG frequency power $\left(\mu V^{2}\right)$ extracted from the wavelet analysis. Coloured bars correspond to first (blue bars), second (red bars) and third (green bars) seconds of the observed video clips intervals were both more effective than the second one $\left(P_{\mathrm{s}}<0.05\right)$ in triggering alpha ERD.

Finally, the analysis of sensory-motor alpha desynchronization in each time interval across conditions showed that during the first $1,000 \mathrm{~ms}$ all observation conditions were equally significantly effective. In the second time interval meaningless movements and grasping were the most effective stimuli $(P<0.05)$ and they did not significantly differ from each other. In the third time interval, similarly to the second one, the most effective stimuli were grasping and meaningless gestures and the latter evoked the strongest sensory-motor alpha ERD $\left(P_{\mathrm{s}}<0.005\right)$.

Time course of observed movement

As shown in Fig. 7, overall, a greater amount of movement was observed during the first 2,000 ms of video clips for all stimuli categories. As far as communicative gestures and meaningless movements were concerned, the hand showed a greater amount of movement during the second compared to the first time-bin of the video clips $(P<0.0001$, $P<0.05)$, but there were no differences in the amount of movement between the second and the third time intervals $(P>0.1, P>0.5)$. On the other hand, the first and the second time-bins in grasping and social grasping conditions were not different from each other, while significantly less amount of movement was revealed during the third compared to the second time interval in both stimuli categories $\left(P_{\mathrm{s}}<0.0001\right)$.

\section{Discussion}

Several previous studies have demonstrated sensory-motor alpha modulation for the observation of goal-related body actions and biological motion (Muthukumaraswamy et al. 2004; Oberman et al. 2007a; Ulloa and Pineda 2007; Babiloni et al. 2009; Perry and Bentin 2009; Southgate et al. 2009, 2010). However, few EEG studies have examined sensory-motor alpha modulation for other kinds of hand stimuli, such as meaningless movements (Babiloni et al. 2002; Lepage and Théoret 2006) and socially-relevant body movements (Kilner et al. 2006; Oberman et al. 2007b).

To the best of our knowledge this is the first study that compares sensory-motor alpha modulation during the observation of different types of hand motor acts and gestures, belonging to different categories on the basis of their goal and social content. Furthermore, this is the first study to integrate the analysis of such diverse stimuli with the analysis of the temporal dynamics of sensory-motor alpha modulation during their observation. 
Fig. 7 Averaged amount of observed hand movement, expressed in $\mathrm{cm}$, in the different stimuli categories. Error bars represent the standard error of the mean. Asterisks indicate statistically significant paired comparisons $(P<0.05)$

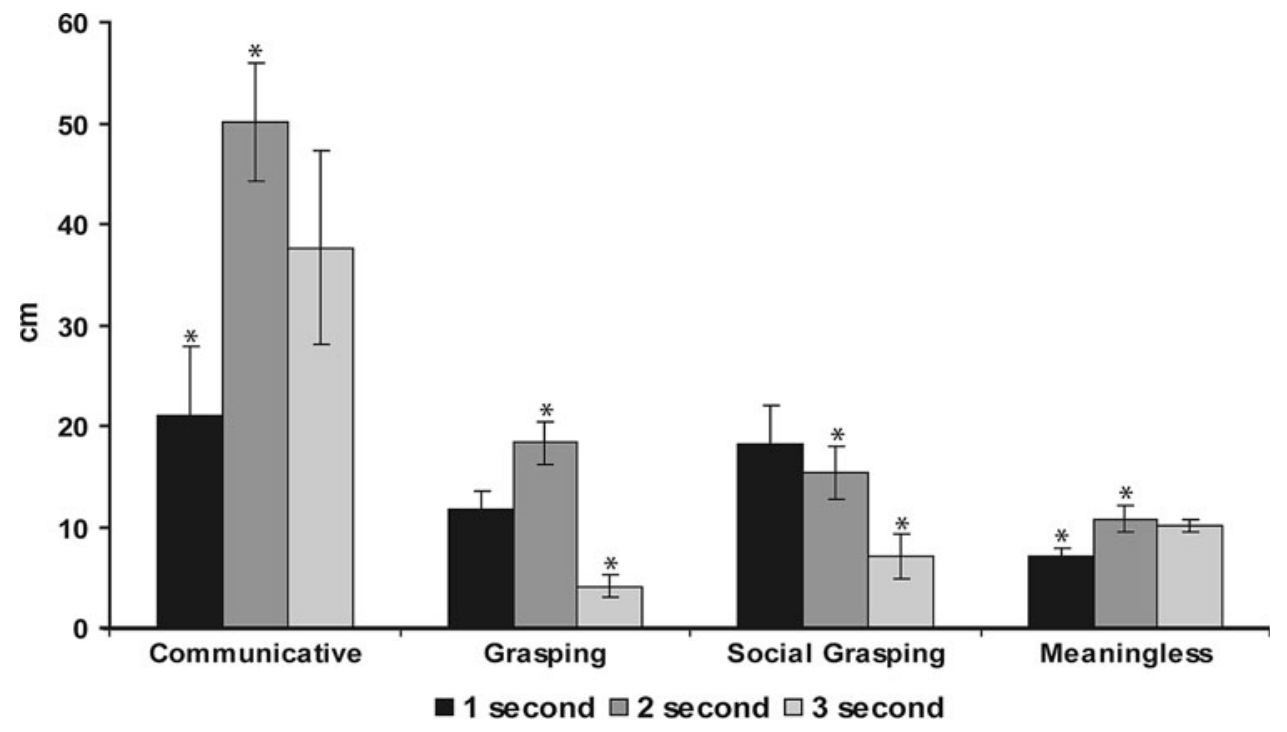

The first result of the present study is that all stimuli were effective in evoking ERD of the cortical sensorymotor region. The fact that both the observation of meaningless hand movements and communicative hand gestures induced ERD modulation is in agreement with the results of previous EEG (Babiloni et al. 2002; Lepage and Théoret 2006), fMRI (Lui et al. 2008; Montgomery et al. 2007) and TMS studies (Fadiga et al. 1995). However, none of those studies systematically compared action execution and observation and applied such analysis to so many different categories of hand motor behaviours as we did here. The present result is important, since it demonstrates, within the same group of participants, the relevance of any observed hand motor behaviour in activating motor resonance in the brain of the observer.

Concerning the lateralization of ERD, our data show bilateral ERD during the observation of all stimuli. This result is in agreement with some previous studies (Babiloni et al. 2002; Muthukumaraswamy et al. 2004).

Concerning the time course analysis of alpha suppression, one important result of the present study is that, in spite of the fact that the observation of all stimuli was effective in triggering the sensory-motor alpha ERD, this effect showed a different time course according to the category of observed hand actions and gestures. The first time interval was the most effective in triggering sensorymotor alpha ERD during stimuli observation, since all stimuli equally evoked alpha suppression during this epoch. Similarly, Babiloni et al. (2002) have found the peak of alpha ERD in the first $1000 \mathrm{~ms}$ during the observation of meaningless movements and, as demonstrated by Orgs et al. (2008), during the observation of dance movements, modulation of alpha ERD was particularly strong within early time intervals of video clips observation.
However, in the present study the different categories of observed stimuli evoked different ERD modulation during the remaining part of the observed video clips. Specifically, the observation of meaningless hand movements induced equal sensory-motor alpha ERD in each of the three time intervals: the modulation started during the first $1,000 \mathrm{~ms}$ and lasted for the whole duration of the video clips. Overall meaningless gestures and hand grasping were the most effective stimuli, and induced the strongest ERD modulation during the second and third time intervals of observation. It has been previously proposed that alpha ERD modulation could depend on the type of task and reflect differences in the cognitive complexity, familiarity and social relevance of stimuli (Klimesch 1999; Klimesch et al. 2007; Oberman et al. 2008; Kilner et al. 2006).

The results of the present study, however, show that such modulation is not equally distributed in time. In fact, ERD is initially equally modulated in spite of differences along the above mentioned dimensions of the stimuli, while shows different modulations with different stimuli during the ensuing time-bins. Such an effect could be either related to the different goals and social dimension of the observed stimuli, or, more trivially, to surface level features like the quantity of observed movement, which do not necessarily vary with action/movement type. To verify this hypothesis we analyzed the quantity of movement during each time interval for the four different conditions of observed hand behaviours. The results of this analysis showed that there was no correlation at all between the quantity of observed movement during the different timebins and ERD amplitude. For example, communicative gestures did not show any significant ERD during the second time-bin. However, the very same epoch was characterized by the highest movement quantity. Conversely, communicative gestures evoked the strongest ERD 
during the first time-bin, in which the quantity of movement was less than in the subsequent epoch. Thus, the equal modulation of ERD during the first $1,000 \mathrm{~ms}$ for all categories of observed hand behaviours is most likely due to the motor resonance that occurs immediately and automatically. The presence of a goal or social value in the observed hand behaviour or its occurrence within a social context are not necessary conditions to evoke motor resonance. Once the hand motor representation of the observed behaviour has been activated in the observer, the intensity and stability of its activation appear to depend upon the goal-relatedness and social relevance of the observed behaviour. Indeed, only communicative gestures and social grasping - sharing both goal-relatedness and social valueshowed a dynamic pattern of ERD modulation during the second and third $1,000 \mathrm{~ms}$ of the video clips presentation.

The observation of social hand behaviours, such as social grasping triggers a more dynamically modulated motor resonance mechanism compared to that driven by the observation of simple grasping. Simple grasping followed by holding of the object evokes a prolonged alpha suppression during the entire duration of its observation, likely reflecting motor resonance occurring during the observation of hand pre-shaping, actual grasping and object holding. Once the most trivial and parsimonious explanation of these dynamic modulations is ruled out (see above), two not mutually exclusive explanations can be proposed for the different ERD temporal modulation evoked by social grasping observation. On the one hand, it has been shown that the kinematics of the same hand motor act differs according to its social or non social nature (Becchio et al. 2008a; Sartori et al. 2009a, b). More specifically, the authors (Becchio et al. 2008b) have found a lower movement amplitude and earlier peak of velocity during the execution of a goal-directed action in social context (giving an object to another individual) than in 'single-agent' condition (placing an object on a base). In other words, deceleration phase was longer (thus action more accurate) when the hand was approaching another individual. It could be proposed that the specific kinematics pattern of an executed social hand action may influence the differences in the temporal pattern of ERD also during the observation of social grasping when this condition is compared with grasping. Thus, the observation of social grasping most likely induces in the observer a different motor resonance compared to the observation of simple grasping. Our data suggest that such difference develops in time. On the other hand, social grasping also appears more ambiguous than simple grasping. While in the latter there is only one acting agent, in social grasping there are two agents, and the observer in principle might not know with whom to resonate, because social grasping potentially implies two different motor acts, offering an object and/or grasping it. It is no coincidence that at debriefing several participants pointed out that with social grasping they were not sure about the goal of the observed motor act, described alternatively as a grasping or as a giving. Our data show that only during the third time interval of video clip presentation, when social grasping is completed with the holding phase, thus when the goal of grasping becomes clear, ERD increases and becomes as strong as during the first $1,000 \mathrm{~ms}$ of observation. Finally, another factor could influence the reduction of ERD in the second time interval of social grasping. As the motor act develops, visual attention is shared between two interacting hands. It is plausible that during the second time interval participants shift their attention to the static hand of a potential 'giver' as they are unsure whether the giver will do something of interest. Although, we cannot provide further elements to this hypothesis, as the eye-tracker was not used in the present study, the attentional account could have strong implications when comparing conditions with one versus two biological stimuli/agents.

Comparing the two types of stimuli within the 'gesture' category, again we observe a clear difference in the temporal pattern of sensory-motor alpha modulation. ERD modulation was more stable for meaningless gestures. With such stimuli, alpha remained desynchronised for the whole period of observation, while for communicative gestures alpha desynchronised during the first 1,000 ms of observation, and resynchronized during the second time epoch, when ERD disappeared with respect to baseline. During the third second of observation, ERD reappeared, although being significantly weaker when compared with meaningless gestures.

We presume that this difference might again depend upon the different goal-relatedness of the observed hand gestures. The observation of meaningless gestures triggers ERD during the whole duration of the stimuli without any time course modulation. The prolonged ERD could be due to an activation of the motor resonance mechanism lasting as long as the hand continues to move, perhaps waiting for a potential goal to emerge. It is possible to hypothesize that when observing hand behaviors devoid of any meaning, humans nevertheless cannot refrain from trying to find a meaning, hence the necessity to keep motor resonance active.

In contrast, during the observation of communicative gestures, motor resonance finishes as soon as the meaning is understood, that is, in our case at the end of the first $1,000 \mathrm{~ms}$ of observation. In agreement with this interpretation, communicative gestures evoke reduced N400 compared to meaningless gestures, and this effect has a centro-posterior distribution (Gunter and Bach 2004). The resumed desynchronization occurring during the last second of observation might be due to the persistence of a static hand, which nevertheless conveys a social meaning, thus inducing motor resonance to be resumed. 
In our view, there are two different functional aspects related to the activation of the mirror mechanism during movement observation in humans. The first one represents an automatic low-level motor resonance, starting as soon as a movement or a goal-related motor act is observed, which triggers eventually the understanding process. Differently from the available evidence in monkeys, in humans motor resonance can be induced also when a motor goal is not present in the observed behavior of others (Fadiga et al. 1995; Babiloni et al. 2002). Most likely, the cortical motor system of humans contains neurons that can be activated by the observation of meaningless movements. Action understanding is the second functional aspect related to the mirror mechanism. Such aspect implies the activation of goal-related motor neurons in the brain of the observer matching the goal of the observed motor behavior of others. For meaningless movements, there is only the first aspect of the mirror mechanism activation. Brain imaging experiments are being designed to investigate the possible segregation of these two different aspects of the mirror mechanism for hand motor behaviors.

In summary, the present study clearly shows that motor resonance can be induced whenever hand motor behavior is observed, irrespective of its goal-relatedness and social content. However, motor resonance is not an all-or nothing mechanism, but can be strongly modulated in time according to-at least-two different dimensions of the observed hand behaviors: the presence/absence of a goal and its social relevance. It is important to emphasize that such differences can be detected, only if one investigates the temporal pattern of the mirror mechanism activation.

Acknowledgments The authors wish to thank Prof. C.A. Tassinari for most valuable comments on EEG data collection and analysis, Dr. Pietro Avanzini for his assistance in wavelet analysis and Dr. Patricia Gough for helpful comments on earlier draft of this manuscript, Dr. Marco Bimbi and Dr. Fabian Chersi for providing the technical support for movement analysis This research was supported by Ministero dell'Università e della Ricerca [Relevant National Interest Project]. A.S. was funded by the Marie Curie Research Training Network "Disorders and Coherence of the Embodied Self". C.B. was supported by fellowship Ricerca Finalizzata 2007-Programma Strategico "Inquiry into disruption of inter-subjective equipment in autistic spectrum disorders in childhood".

Open Access This article is distributed under the terms of the Creative Commons Attribution Noncommercial License which permits any noncommercial use, distribution, and reproduction in any medium, provided the original author(s) and source are credited.

\section{References}

Altschuler EL, Vankov A, Wang V, Ramachandran VS, Pineda JA. (1997) Person see, person do: human cortical electrophysiological correlates of monkey see monkey do cell. Soc Neurosci 719 (17)
Altschuler EL, Vankov A, Hubbard EM, Roberts E, Ramachandran VS, Pineda JA (2000) Mu wave blocking by observation of movement and its possible use as a tool to study theory of other minds. Soc Neurosci 68 (1)

Aziz-Zadeh L, Wilson SM, Rizzolatti G, Iacoboni M (2006) Congruent embodied representations for visually presented actions and linguistic phrases describing actions. Curr Biol 16(18):1818-1823

Babiloni C, Babiloni F, Carducci F, Cincotti F, Cocozza G, Del Percio C, Moretti DV, Rossini PM (2002) Human cortical electroencephalography (EEG) rhythms during the observation of simple aimless movements: a high resolution EEG study. Neuroimage 17:559-572

Babiloni C, Del Percio C, Rossini PM, Marzano N, Iacoboni M, Infarinato F, Lizio R, Piazza M, Pirritano M, Berlutti G, Cibelli G, Eusebi F (2009) Judgment of actions in experts: a high-resolution EEG study in elite athletes. Neuroimage 45(2):512-521

Becchio C, Sartori L, Bulgheroni M, Castiello U (2008a) Both your intention and mine are reflected in the kinematics of my reachto-grasp movement. Cognition 106(2):894-912

Becchio C, Sartori L, Bulgheroni M, Castiello U (2008b) The case of Dr. Jekyll and Mr. Hyde: a kinematic study on social intention. Conscious Cogn 17(3):557-564

Bernier R, Dawson G, Webb S, Murias M (2007) EEG mu rhythm and imitation impairments in individuals with autism spectrum disorder. Brain Cogn 64:228-237

Buccino G, Binkofski F, Fink GR, Fadiga L, Fogassi L et al (2001) Action observation activates premotor and parietal areas in a somatotopic manner: an fMRI study. Eur J Neurosci 13:400-404

Calmels C, Holmes P, Jarry G, Lévèque JM, Hars M, Stam CJ (2006) Cortical activity prior to, and during, observation and execution of sequential finger movements. Brain Topogr 19(1-2):77-88

Cattaneo L, Rizzolatti G (2009) The mirror neuron system. Review. Arch Neurol 66(5):557-560

Cochin S, Barthelemy C, Lejeune B, Roux S, Martineau J (1998) Perception of motion and qEEG activity in human adults. Electroencephalogr Clin Neurophysiol 107:287-295

Cochin S, Barthelemy C, Roux S, Martineau J (1999) Observation and execution of movement: similarities demonstrated by quantified electroencephalograpy. Eur J Neurosci 11:1839-1842

Cohen-Seat G, Gastaut H, Faure J, Heuyer G (1954) Etudes experiementales de l'activité nerveuse pendant la projection cinematographique. Rev Int de Filmologie 5:7-64

Di Pellegrino G, Fadiga L, Fogassi L, Gallese V, Rizzolatti G (1992) Understanding motor events: a neurophysiological study. Exp Brain Res 91:176-180

Fadiga L, Fogassi L, Pavesi G, Rizzolatti G (1995) Motor facilitation during action observation: a magnetic stimulation study. J Neurophysiol 73:2608-2611

Gallese V, Fadiga L, Fogassi L, Rizzolatti G (1996) Action recognition in the premotor cortex. Brain 119:593-609

Gallese V, Keysers C, Rizzolatti G (2004) A unifying view of the basis of social cognition. Trends Cogn Sci 8(9):396-403

Gastaut HJ, Bert J (1954) EEG changes during cinematographic presentation, moving picture activation of the EEG. Electroencephalogr Clin Neurophysiol 6:433-444

Gazzola V, Aziz-Zadeh L, Keysers C (2006) Empathy and the somatotopic auditory mirror system in human. Curr Biol 16:1824-1829

Gunter TC, Bach P (2004) Communicating hands: ERPs elicited by meaningful symbolic hand postures. Neurosci Lett 372(1-2):52-56

Hari R (2006) Action-perception connection and the cortical mu rhythm. Prog Brain Res 159:253-260

Hari R, Salmelin R (1997) Human cortical oscillations: a neuromagnetic view through the skull. Trends Neurosci 20(1):44-49 
Hari R, Forss N, Avikainen S, Kirveskari S, Salenius S, Rizzolatti G (1998) Activation of human primary motor cortex during action observation: a neuromagnetic study. Proc Natl Acad Sci USA 95:15061-15065

Kilner JM, Marchant JL, Frith CD (2006) Modulation of the mirror system by social relevance. Soc Cogn Affect Neurosci 1(2):143-148

Klimesch W (1999) EEG alpha and theta oscillations reflect cognitive and memory performance: a review and analysis. Brain Res Rev 29:169-195

Klimesch W, Sauseng P, Hanslmayr S (2007) EEG alpha oscillations: the inhibition-timing hypothesis. Brain Res Rev 53:63-88

Lepage JF, Théoret H (2006) EEG evidence for the presence of an action observation-execution matching system in children. Eur $\mathrm{J}$ Neurosci 23(9):2505-2510

Lui F, Buccino G, Duzzi D, Benuzzi F, Crisi G, Baraldi P, Nichelli P, Porro CA (2008) Neural substrates of observing and imaging non-object directed action. Soc Neurosci 3:261-275

Montgomery KJ, Isenberg N, Haxby JV (2007) Communicative hand gestures and object-directed hand movements activated the mirror neuron system. Soc Cogn Affect Neurosci 2(2):114-122

Mukamel R, Ekstrom AD, Kaplan J, Iacoboni M, Fried I (2010) Single-neuron responses in humans during execution and observation of actions. Curr Biol 20(8):750-756

Muthukumaraswamy SD, Johnson BW (2004a) Changes in rolandic mu rhythm during observation of a precision grip. Psychophysiolgy 41:152-156

Muthukumaraswamy SD, Johnson BW (2004b) Primary motor cortex activation during action observation revealed by wavelet analysis of the EEG. Clin Neurophysiol 115:1760-1766

Muthukumaraswamy SD, Johnson BW, McNair NA (2004) Mu rhythm modulation during observation of an object-directed grasp. Cogn Brain Res 19:195-201

Nakamura K, Kawashima R, Ito K, Sugiura M, Kato T, Nakamura A, Hatano K, Nagumo S, Kubota K, Fukuda H, Kojima S (1999) Activation of the right inferior frontal cortex during assessment of facial emotion. J Neurophysiol 82:1610-1614

Nakamura A, Maess B, Knösche TR, Gunter TC, Bach P, Friederici $A D$ (2004) Cooperation of different neuronal systems during hand sign recognition. Neuroimage 23(1):25-34

Oberman LM, McCleery JP, Ramachandran VS, Pineda JA (2007a) EEG evidence for mirror neuron activity during the observation of human and robot actions: toward an analysis of the human qualities of interactive robots. Neurocomputing 70:2194-2203

Oberman LM, Pineda JA, Ramachandran VS (2007b) The human mirror neuron system: a link between action observation and social skills. Soc Cogn Affect Neurosci 6:62-66
Oberman LM, Ramachandran VS, Pineda JA (2008) Modulation of mu suppression in children with autism spectrum disorders in response to familiar or unfamiliar stimuli: the mirror neuron hypothesis. Neuropsychology 46(5):1558-1565

Oldfield RC (1971) The assessment and analysis of handedness: the Edinburgh Inventory. Neuropsychology 9:77-113

Orgs G, Dombrowski JH, Heil M, Jansen-Osmann P (2008) Expertise in dance modulates alpha/beta event-related desynchronization during action observation. Eur J Neurosci 27:3380-3384

Perry A, Bentin S (2009) Mirror activity in the human brain while observing hand movements: a comparison between EEG desynchronization in the mu-range and previous fMRI results. Brain Res 1282:126-132

Pfurtscheller G, Lopes da Silva FH (1999) Event-related EEG/MEG synchronization and desynchronization: basic principles. Clin Neurophysiol 110:1842-1857

Pineda JA (2005) The functional significance of mu rhythms: translating "seeing" and "hearing" into "doing". Brain Res Rev 50:57-68

Rizzolatti G, Fadiga L, Gallese V, Fogassi L (1996) Premotor cortex and the recognition of motor actions. Cogn Brain Res 3:131-141

Rizzolatti G, Craighero L (2004) The mirror-neuron system. Annu Rev Neurosci 27:169-192

Salmelin R, Hari R (1994) Spatiotemporal characteristics of sensorimotor neuromagnetic rhythms related to thumb movement. Neuroscience 60:537-550

Sartori L, Becchio C, Bulgheroni M, Castiello U (2009a) Modulation of the action control system by social intention: unexpected social requests override preplanned action. J Exp Psychol Hum Percept Perform 35(5):1490-1500

Sartori L, Becchio C, Bara BG, Castiello U (2009b) Does the intention to communicate affect action kinematics? Conscious $\operatorname{Cog} 18(3): 766-772$

Southgate V, Johnson MH, Osborne T, Csibra G (2009) Predictive motor activation during action observation in human infants. Biol Lett 5(6):769-772

Southgate V, Johnson MH, El Karoui I, Csibra G (2010) Motor system activation reveals infants' on-line prediction of others' goals. Psychol Sci 21(3):355-359

Ulloa ER, Pineda JA (2007) Recognition of point-light biological motion: mu rhythms and mirror neuron activity. Behav Brain Res 183(2):188-194

Villarreal M, Fridman EA, Amengual A, Falasco G, Gerscovich ER, Ulloa ER, Leiguarda RC (2008) The neural substrate of gesture recognition. Neuropsychology 46(9):2371-2382 